J. Korean Math. Soc. 49 (2012), No. 2, pp. 357-378

http://dx.doi.org/10.4134/JKMS.2012.49.2.357

\title{
THE EXISTENCE OF GLOBAL ATTRACTOR FOR CONVECTIVE CAHN-HILLIARD EQUATION
}

\author{
Xiaopeng Zhao and Bo LiU
}

\begin{abstract}
In this paper, we consider the convective Cahn-Hilliard equation. Based on the regularity estimates for the semigroups, iteration technique and the classical existence theorem of global attractors, we prove that the convective Cahn-Hilliard equation possesses a global attractor in $H^{k}(k \geq 0)$ space, which attracts any bounded subset of $H^{k}(\Omega)$ in the $H^{k}$-norm.
\end{abstract}

\section{Introduction}

In this paper, we consider the existence of global attractor for the convective Cahn-Hilliard equation

$$
\frac{\partial u}{\partial t}+\gamma \Delta^{2} u=\Delta A(u)+\beta \cdot \nabla B(u), \quad x \in \Omega,
$$

where $\Omega$ is a bounded domain in $\mathbb{R}^{n}(n \leq 2)$. On the basis of physical considerations, as usual Eq.(1.1) is supplemented with the following boundary value conditions

$$
u(x, t)=\Delta u(x, t)=0, \quad x \in \partial \Omega,
$$

and the initial value condition

$$
u(x, 0)=u_{0}(x) .
$$

Eq.(1.1) arises naturally as a continuous model for the formation of facets and corners in crystal growth, see $[5,17]$. Here $u(x, t)$ denotes the slope of the interface. The convective term $\beta \cdot \nabla B(u)$, see [5], stems from the effect of kinetic that provides an independent flux of the order parameter, similar to the effect of an external field in spinodal decomposition of a driven system. For small driving force $\beta \rightarrow 0$, Eq.(1.1) is reduced to the well-known Cahn-Hilliard equation.

During the past years, only a few papers were devoted to the convective Cahn-Hilliard equation. It was K. H. Kwek [6] who first studied the convective

Received December 4, 2010.

2010 Mathematics Subject Classification. 35B41, 35K35.

Key words and phrases. attractor, convective Cahn-Hilliard equation, absorbing set.

(C)2012 The Korean Mathematical Society 
Cahn-Hilliard equation (1.1) for the case with convection, namely, $B(u)=u$. By some a priori estimates, he proved the existence of a classical solution, and gave the error estimates by the discontinuous Galerkin method. A. Podolny et al. [12] studied the convective Cahn-Hilliard equation with $A(u)=u^{3}-u$ and $B(u)=u^{2}$. In their paper, the dynamics of domain walls (kinks) governed by the convective Cahn-Hilliard equation was studied by means of asymptotic and numerical methods. M. A. Zaks et al. [19] investigate bifurcations of stations periodic solutions of a convective Cahn-Hilliard equation, they described phase separation in driven systems, and studied the stability of the main family of these solutions. Eden and Kalantarov [3, 4] considered the convective Cahn-Hilliard equation as [12] with periodic boundary conditions in one space dimension and three space dimension. They established some result on the existence of a compact attractor. Recently, X. Zhao and C. Liu [20] studied the equation (1.1) with $A(u)=\gamma_{2} u^{3}+\gamma_{1} u^{2}-u$ and $B(u)=-\frac{1}{4} u^{4}+\frac{1}{2} u^{2}$ in two space dimension. They used a classical theorem on the existence of global attractor to derive that the equation (1.1) possesses a global attractor on some subset of $H^{2}$. There is much literature concerned with the convective CahnHilliard equation, for more recent results we refer the reader to $[8,9,17]$ and the references therein.

The dynamic properties of convective Cahn-Hilliard equations such as the global asymptotical behaviors of solutions and global attractors are important for the study of convective Cahn-Hilliard system, which ensure the stability of parabolic phenomena and provide the mathematical foundation for the study of parabolic dynamics. So in this paper we are interested in the existence of global attractors for Eq.(1.1). We shall use the regularity estimates for the linear semigroups, combining with the iteration technique and the classical existence theorem of global attractors, to prove that the problem (1.1)-(1.3) possesses a global attractor in $H^{k}(k \geq 0)$ space.

This paper is organized as follows. In Section 2, based on R. Temam's classical theorem, we shall prove the result on the existence of global attractor in $H^{2}$ space. In Section 3, using the theorem on the global attractor of $H^{2}$ space, the existence of global attractors for problem (1.1)-(1.3) in $H^{k}(k \geq 0)$ space is proved. Our important ideas come from $[1,2,7,10,13,14,15,18]$, etc.

Throughout this paper, we denote $L^{2}, L^{p}$ and $H^{k}(k=1,2,3)$ norm in $\Omega$ simply by $\|\cdot\|,\|\cdot\|_{p}$ and $\|\cdot\|_{H^{k}}$. In the following, $C, C_{i}(i=1,2, \ldots)$ will represent generic positive constants that may change from line to line even if in the same inequality.

\section{Attractor in $H^{2}$ space}

\subsection{Preliminary}

Assume $X$ and $X_{1}$ are two Banach spaces, $X_{1} \subset X$ a compact and dense inclusion. Consider the following equation defined on $X$, 


$$
\begin{cases}u_{t} & =L u+G(u) \\ u(0) & =\varphi\end{cases}
$$

where $u$ is an unknown function, $L: X_{1} \rightarrow X$ a linear operator and $G: X_{1} \rightarrow X$ a nonlinear operator. Then the solution of (2.1) can be expressed as

$$
u(t, \varphi)=S(t) \varphi,
$$

where $S(t): X \rightarrow X(t \geq 0)$ is a semigroup generated by (2.1).

For convenience, we recall some basic concepts on infinite dimensional dynamical systems.

Definition 2.1. Suppose $S(t)$ is an operator semigroup defined on $H$, A set $\Sigma \subset H$ is called an invariant set of $S(t)$ if $\forall t \geq 0, S(t) \Sigma=\Sigma$. An invariant set is called an attractor of $S(t)$ if $\Sigma$ is compact, and there exists a neighborhood $U \subset H$ of $\Sigma$ such that for any $\varphi \in U$,

$$
\operatorname{dist}(S(t) \varphi, \Sigma)=\inf _{v \in \Sigma}\|S(t) \varphi-v\|_{H} \rightarrow 0 \text { as } t \rightarrow \infty .
$$

Therefore, we can say $\Sigma$ attracts $U$. In particular, if $\Sigma$ attracts any bounded subset of $H, \Sigma$ is a global attractor.

For a set $D \subset H$, the $\omega$-limit set of $D$ is defined by

$$
\omega(D)=\bigcap_{s \geq 0} \overline{\bigcup_{t \geq s} S(t) D}
$$

where the closure is taken in the $H$-norm.

Next, we introduce the classical existence theorem of global attractor by R. Temam [16].

Lemma 2.1. Assume that $S(t): X \rightarrow X$ is the semigroup generated by problem (2.1), and the following conditions hold:

(H1) For any bounded set $A \subset X$ there exists a time $t_{A} \geq 0, \forall \varphi \in A, t>t_{A}$, we have $S(t) \varphi \in B$;

(H2) For any bounded set $u \subset X$ and some $T>0$ sufficiently large, the set $\overline{\bigcup_{t>T} S(t) u}$ is compact in $X$.

Then the $\omega$-limit set $\mathcal{A}=\omega(B)$ of $B$ is a global attractor of problem (2.1), and $\mathcal{A}$ is connected providing $B$ is connected.

In this paper, the function $A(u)$ is a polynomial of order $2 p-1$

$$
A(u)=\sum_{i=1}^{2 p-1} a_{i} u^{i}, p \in \mathbb{N}, p \leq \frac{2}{n}+1,
$$

with leading coefficient $a_{2 p-1}>0$, the function $B(u)$ is also a polynomial, with the order $q$

$$
B(u)=\sum_{j=1}^{q} b_{j} u^{j}, q \in \mathbb{N}, q \leq \frac{6}{n}+1 .
$$


Thus, we introduce the lemma on global existence and uniqueness for the solution of problem (1.1)-(1.3).

Lemma 2.2. Suppose that $u_{0} \in H_{0}^{2}(\Omega)$, Then problem (1.1)-(1.3) admits a global weak solution $u \in H_{0}^{2}(\Omega)$.

We can obtained Lemma 2.2 by the standard Galerkin methods. Since the proof is so standard, we omit it.

By Lemma 2.2, we can define the operator semigroup $\{S(t)\}_{t \geq 0}$ in $H^{2}$ space as

$$
S(t) u_{0}=u(t), \forall u_{0} \in H_{0}^{2}(\Omega), t \geq 0,
$$

where $u(t)$ is the solution of (1.1)-(1.3) corresponding to initial value $u_{0}$. We also get the operator semigroup $\{S(t)\}_{t \geq 0} u_{0}$ by solution $u$ is strongly continuous.

To study the existence of global attractor, we have to find a closed metric space and prove that there exists a global attractor in the closed metric space. Notice that it is alright to consider the global existence and uniqueness of the solution of the problem (1.1)-(1.3) for any given initial datum, as described in the previous lemma. However, because $\left\{u \mid u \in H_{0}^{2}(\Omega)\right\}$ is a closed metric space, we let $\mathscr{U}=H_{0}^{2}(\Omega)$. It is easy to see that the restriction of $\{S(t)\}$ on the affined space $\mathscr{U}$ is a well defined semigroup.

Then, we give the main result of this section:

Theorem 2.1. Suppose that $\Omega$ denote an open bounded domain in $\mathbb{R}^{n}(n \leq 2)$. Then for every a chosen as above, the semiflow associated with the solution $u$ of the problem (1.1)-(1.3) possesses in $\mathscr{U}$ a global attractor $\mathscr{A}$ which attracts all the bounded set in $\mathscr{U}$.

\subsection{Proof of Theorem 2.1}

In order to prove Theorem 2.1, we establish some a priori estimates for the solution $u$ of problem (1.1)-(1.3). In this part we always assume that $\{S(t)\}_{t \geq 0}$ is the semigroup generated by the weak solutions of problem (1.1)-(1.3) with initial data $u_{0} \in H^{2}(\Omega)$. Thus, we have the following lemma.

Lemma 2.3. There exists a bounded set $\mathscr{B}$ whose size depends only on $\Omega$, in $\mathscr{U}$ such that for all the orbits starting from any bounded set $B$ in $\mathscr{U}, \exists t_{0}=$ $t_{0}(B) \geq 0$ s.t. $\forall t \geq t_{0}$ all the orbits will enter and remain in $\mathscr{B}$.

Proof. It suffices to prove that there is a positive constant $C$ such that

$$
\|u(t)\|_{H^{2}} \leq C, \quad t \geq t_{0}(B) .
$$

We prove the lemma in the following steps.

Step 1: $L^{2}$ norm estimate. Multiplying (1.1) with $u$, and integrating the result over $\Omega$, we deduce that

$$
\frac{1}{2} \frac{d}{d t}\|u\|^{2}+\gamma\|\Delta u\|^{2}=(\Delta A(u), u)+(\beta \cdot \nabla B(u), u) .
$$


Hence

$$
\frac{1}{2} \frac{d}{d t}\|u\|^{2}+\gamma\|\Delta u\|^{2}=-\int_{\Omega} A^{\prime}(u)|\nabla u|^{2} d x-\beta \cdot \int_{\Omega} B(u) \nabla u d x .
$$

A simple calculation shows that

$$
A^{\prime}(u)=(2 p-1) a_{2 p-1} u^{2 p-2}+(2 p-2) a_{2 p-2} u^{2 p-3}+\cdots+a_{2} u+a_{1}
$$

$$
\geq k_{1} u^{2 p-2}-k_{2}
$$

where $k_{1}$ and $k_{2}$ are positive constants. We also have

$$
(\beta \cdot \nabla B(u), u)=\int_{\Omega} \beta \cdot(\nabla B(u)) u d x=0 .
$$

Adding (2.4)-(2.6) together, we get

$$
\frac{1}{2} \frac{d}{d t}\|u\|^{2}+\gamma\|\Delta u\|^{2}+k_{1} \int_{\Omega} u^{2 p-2}|\nabla u|^{2} d x \leq k_{2} \int_{\Omega}|\nabla u|^{2} d x .
$$

Thus

$$
\frac{1}{2} \frac{d}{d t}\|u\|^{2}+\gamma\|\Delta u\|^{2} \leq k_{2}\|\nabla u\|^{2} \leq k_{2}\|u\| \cdot\|\Delta u\| \leq \frac{\gamma}{2}\|\Delta u\|^{2}+\frac{k_{2}^{2}}{2 \gamma}\|u\|^{2},
$$

which means

$$
\frac{d}{d t}\|u\|^{2}+\gamma\|\Delta u\|^{2} \leq \frac{k_{2}^{2}}{\gamma}\|u\|^{2}
$$

On the other hand, by Poincaré's inequality for function in $\mathscr{U}$, we have

$$
\|u\|^{2} \leq c_{1}\|\nabla u\|^{2}
$$

where $c_{1}$ depends only on the domain. In addition, we also have the following inequality

Hence

$$
c_{1}\|\nabla u\|^{2} \leq c_{1}\|u\| \cdot\|\Delta u\| \leq \frac{1}{2}\|u\|^{2}+\frac{c_{1}^{2}}{2}\|\Delta u\|^{2} .
$$

$$
\|u\|^{2} \leq c_{1}^{2}\|\Delta u\|^{2}
$$

Adding (2.8) and the above inequality together, we finally arrive at

$$
\frac{d}{d t}\|u\|^{2}+\left(\frac{\gamma}{c_{1}^{2}}-\frac{k_{2}^{2}}{\gamma}\right)\|u\|^{2} \leq 0
$$

Taking $\gamma$ large enough, satisfies $\frac{\gamma}{c_{1}^{2}}-\frac{k_{2}^{2}}{\gamma}>0$, we obtain

$$
\|u\|^{2} \leq e^{-\left(\frac{\gamma}{c_{1}^{2}}-\frac{k_{2}^{2}}{\gamma}\right) t}\left\|u_{0}\right\|^{2} .
$$

Thus, for initial data in any bounded set $B \subset \mathscr{U}$, there is a uniform time $t_{1}(B)$ depending on $B$ such that for $t \geq t_{1}(B)$, there holds

$$
\|u(x, t)\|^{2} \leq C_{1} \text {. }
$$


Step 2: $H^{1}$ norm estimate. Multiplying (1.1) with $\Delta u$, and integrating the result over $\Omega$, we obtain

(2.11) $\quad \frac{1}{2} \frac{d}{d t}\|\nabla u\|^{2}+\gamma\|\nabla \Delta u\|^{2}=-\int_{\Omega} \Delta A(u) \Delta u d x-\beta \cdot \int_{\Omega} \nabla B(u) \Delta u d x$.

Note that

$$
\Delta A(u)=A^{\prime \prime}(u)(\nabla u)^{2}+A^{\prime}(u) \Delta u .
$$

A simple conclusion shows that

$$
\left|A^{\prime}(u)\right| \leq k_{3}\left(1+|u|^{2 p-2}\right),\left|A^{\prime \prime}(u)\right| \leq k_{4}\left(1+|u|^{2 p-3}\right),
$$

we also have

$$
B(u)=\sum_{j=1}^{q} b_{j} u^{j} \leq k_{5} u^{q}+k_{6},
$$

where $k_{3}, k_{4}, k_{5}, k_{6}$ are positive constants. By (2.5), (2.11)-(2.13), we get

$$
\begin{aligned}
& \frac{1}{2} \frac{d}{d t}\|\nabla u\|^{2}+\gamma\|\nabla \Delta u\|^{2}+k_{1} \int_{\Omega} u^{2 p-2}|\Delta u|^{2} d x \\
\leq & k_{4} \int_{\Omega}\left(1+|u|^{2 p-3}\right)|\nabla u|^{2} \Delta u d x+k_{2}\|\Delta u\|^{2} \\
& +k_{5}|\beta| \int_{\Omega}\left|u^{q} \nabla \Delta u\right| d x+k_{6}|\beta| \int_{\Omega}|\nabla \Delta u| d x \\
\leq & \frac{k_{4}}{2}\|\nabla u\|_{4}^{4}+\left(\frac{k_{4}}{2}+k_{2}\right)\|\Delta u\|^{2}+k_{1} \int_{\Omega} u^{2 p-2}(\Delta u)^{2} d x \\
& +\frac{k_{4}^{2}}{4 k_{1}} \int_{\Omega} u^{2 p-4}(\nabla u)^{4} d x+\frac{\gamma}{7}\|\nabla \Delta u\|^{2} \\
& +\frac{7 k_{5}|\beta|}{2 \gamma}\|u\|_{2 q}^{2 q}+\frac{7 k_{6}|\beta|}{2 \gamma}|\Omega| .
\end{aligned}
$$

Thus,

$$
\begin{aligned}
& \frac{1}{2} \frac{d}{d t}\|\nabla u\|^{2}+\frac{6}{7} \gamma\|\nabla \Delta u\|^{2} \\
\leq & \frac{k_{4}}{2}\|\nabla u\|_{4}^{4}+\left(\frac{k_{4}}{2}+k_{2}\right)\|\Delta u\|^{2}+\frac{k_{4}^{2}}{4 k_{1}} \int_{\Omega} u^{2 p-4}(\nabla u)^{4} d x \\
& +\frac{7 k_{5}|\beta|}{2 \gamma}\|u\|_{2 q}^{2 q}+\frac{7 k_{6}|\beta|}{2 \gamma}|\Omega| \\
= & E_{1}+E_{2}+E_{3}+E_{4}+\frac{7 k_{6}|\beta|}{2 \gamma}|\Omega| .
\end{aligned}
$$

For $E_{1}$, based on Nirenberg's inequality, we derive that

$$
\begin{aligned}
\|\nabla u\|_{4} & \leq M_{1}\|\nabla \Delta u\|^{\frac{1}{3}+\frac{n}{12}}\|u\|^{\frac{2}{3}-\frac{n}{12}}+M_{2}\|u\| \\
& \leq M_{3}\|\nabla \Delta u\|^{\frac{1}{3}+\frac{n}{12}}+M_{4}(n \leq 2),
\end{aligned}
$$


where $M_{i}(i=1,2,3,4)$ here and in the following are positive constants. Thus,

$$
E_{1}=\frac{k_{4}}{2}\|\nabla u\|_{4}^{4} \leq \frac{\gamma}{14}\|\nabla \Delta u\|^{2}+C_{2}
$$

For $E_{2}$, by (2.10), we get

$$
\begin{aligned}
& \int_{\Omega}|\nabla u|^{2} d x=-\int_{\Omega} u \cdot \Delta u d x \\
\leq & \left(\int_{\Omega} u^{2} d x\right)^{\frac{1}{2}}\left(\int_{\Omega}|\Delta u|^{2} d x\right)^{\frac{1}{2}} \leq C\left(\int_{\Omega}|\Delta u|^{2} d x\right)^{\frac{1}{2}},
\end{aligned}
$$

we also have

$$
\int_{\Omega}|\Delta u|^{2} d x=-\int_{\Omega} \nabla u \cdot \nabla \Delta u d x \leq\left(\int_{\Omega}|\nabla u|^{2} d x\right)^{\frac{1}{2}}\left(\int_{\Omega}|\nabla \Delta u|^{2} d x\right)^{\frac{1}{2}}
$$

Hence

$$
\begin{gathered}
E_{2}=\left(\frac{k_{4}}{2}+k_{2}\right) \int_{\Omega}|\Delta u|^{2} d x \\
\leq\left(\frac{k_{4}}{2}+k_{2}\right)\left(\int_{\Omega}|\nabla \Delta u|^{2} d x\right)^{\frac{2}{3}} \leq \frac{\gamma}{14}\|\nabla \Delta u\|^{2}+C_{3} .
\end{gathered}
$$

In order to estimate $E_{3}$, we first estimate $\|u\|_{L^{\infty}}$, we have

$$
\begin{aligned}
\sup |u| & \leq M_{1}\|\nabla \Delta u\|^{\frac{n}{6}}\|u\|^{1-\frac{n}{6}}+M_{2}\|u\| \\
& \leq M_{3}\|\nabla \Delta u\|^{\frac{n}{6}}+M_{4}(n \leq 2) .
\end{aligned}
$$

Hence, using (2.16) and the above inequality, we finally arrive at

$$
\begin{aligned}
E_{3} & =\frac{k_{4}^{2}}{4 k_{1}} \int_{\Omega} u^{2 p-4}(\nabla u)^{4} d x \\
& \leq \frac{k_{4}^{2}}{4 k_{1}}(\sup |u|)^{2 p-4} \int_{\Omega}|\nabla u|^{4} d x \\
& \leq \frac{k_{4}^{2}}{4 k_{1}}\left(M_{3}\|\nabla \Delta u\|^{\frac{n}{6}}+M_{4}\right)^{2 p-4}\left(M_{3}\|\nabla \Delta u\|^{\frac{1}{3}+\frac{n}{12}}+M_{4}\right)^{4} \\
& \leq M_{5}\|\nabla \Delta u\|^{\frac{n(p-2)}{3}+\frac{4}{3}+\frac{n}{3}}+M_{6} \\
& \leq \frac{\gamma}{14}\|\nabla \Delta u\|^{2}+C_{4}(n \leq 2) .
\end{aligned}
$$

For $E_{4}$, since

$$
\|u\|_{2 q} \leq M_{1}\|\nabla \Delta u\|^{\frac{n(q-1)}{6 q}}\|u\|^{1-\frac{n(q-1)}{6 q}}+M_{2}\|u\|(n \leq 2),
$$

we have

$$
E_{4}=\frac{7 k_{5}|\beta|}{2 \gamma}\|u\|_{2 q}^{2 q} \leq \frac{\gamma}{7}\|\nabla \Delta u\|^{2}+C_{5}
$$


Adding (2.15), (2.17), (2.20)-(2.22) together, we finally arrive at

$$
\frac{d}{d t}\|\nabla u\|^{2}+\gamma\|\nabla \Delta u\|^{2} \leq 2\left(C_{2}+C_{3}+C_{4}+C_{5}\right)+\frac{7 k_{6}|\beta|}{\gamma}|\Omega| .
$$

Based on (2.18) and (2.19), we also obtain

$$
\|\nabla u\|^{2} \leq C\|\nabla \Delta u\|^{\frac{2}{3}} \leq C_{1}^{\prime}\|\nabla \Delta u\|^{2}+C_{2}^{\prime},
$$

where $C_{1}^{\prime}$ and $C_{2}^{\prime}$ are positive constants. By (2.23) and the above inequality, we deduce that

$$
\frac{d}{d t}\|\nabla u\|^{2}+\frac{\gamma}{C_{1}^{\prime}}\|\nabla u\|^{2} \leq C_{6}
$$

where $C_{6}=2\left(C_{2}+C_{3}+C_{4}+C_{5}\right)+\frac{7 k_{6}|\beta|}{\gamma}|\Omega|+\frac{C_{2}^{\prime} \gamma}{C_{1}^{\prime}}>0$. Using Gronwall's inequality, we deduce that

$$
\|u\|^{2} \leq e^{-\frac{\gamma}{C_{1}^{\prime}} t}\left\|\nabla u_{0}\right\|^{2}+\frac{C_{6} C_{1}^{\prime}}{\gamma} .
$$

Thus, for initial data in any bounded set $B \subset \mathscr{U}$, there is a uniform time $t_{2}(B)$ depending on $B$ such that for $t \geq t_{2}(B)$, there holds

$$
\|\nabla u(x, t)\|^{2} \leq \frac{2 C_{6} C_{1}^{\prime}}{\gamma} .
$$

Adding (2.10) and (2.25) together, we have

$$
\|u(t)\|_{H^{1}} \leq C
$$

By Sobolev's imbedding theorem, we obtain the $L^{m}$ estimate of the function in the space $\mathscr{U}$,

$$
\|u(t)\|_{m} \leq C, m \in \mathbb{N}, m<\infty .
$$

Step 3: $H^{2}$ norm estimate. Multiplying (1.1) by $\Delta^{2} u$, and integrating the result over $\Omega$, we obtain

$$
\frac{1}{2} \frac{d}{d t}\|\Delta u\|^{2}+\gamma\left\|\Delta^{2} u\right\|^{2}=\int_{\Omega} \Delta^{2} u \Delta A(u) d x+\beta \cdot \int_{\Omega} \Delta^{2} u \nabla B(u) d x .
$$

Therefore

$$
\frac{1}{2} \frac{d}{d t}\|\Delta u\|^{2}+\gamma\left\|\Delta^{2} u\right\|^{2} \leq C_{7}\|\Delta A(u)\|^{2}+C_{8}\|\nabla B(u)\|^{2}+\frac{\gamma}{2}\left\|\Delta^{2} u\right\|^{2},
$$

which means

$$
\frac{d}{d t}\|\Delta u\|^{2}+\gamma\left\|\Delta^{2} u\right\|^{2} \leq 2 C_{7}\|\Delta A(u)\|^{2}+2 C_{8}\|\nabla B(u)\|^{2} .
$$

On the other hand, we have

(2.29) $\|\Delta A(u)\|^{2}$

$$
\leq 2\left(\int_{\Omega}\left|A^{\prime}(u)\right|^{2}|\Delta u|^{2} d x+\int_{\Omega}\left|A^{\prime \prime}(u)\right|^{2}|\nabla u|^{4} d x\right)
$$




$$
\begin{aligned}
& \leq 2\left[\left(\int_{\Omega}\left|A^{\prime}(u)\right|^{3} d x\right)^{\frac{2}{3}}\left(\int_{\Omega}|\Delta u|^{6} d x\right)^{\frac{1}{3}}+\left(\int_{\Omega}\left|A^{\prime \prime}(u)\right|^{6} d x\right)^{\frac{1}{3}}\left(\int_{\Omega}|\nabla u|^{6} d x\right)^{\frac{2}{3}}\right] \\
& \leq M_{1}\left[\left(\int_{\Omega}|\Delta u|^{6} d x\right)^{\frac{1}{3}}+\left(\int_{\Omega}|\nabla u|^{6} d x\right)^{\frac{2}{3}}\right],
\end{aligned}
$$

and for $B(u)$, we have

$$
\left|B(u)^{\prime}\right|^{2}=\left(\sum_{j=1}^{q} j b_{j} u^{j-1}\right)^{2} \leq k_{7} u^{2 q-2}+k_{8},
$$

where $k_{7}$ and $k_{8}$ are positive constants. Hence

$$
\begin{aligned}
\|\nabla B(u)\|^{2} & \leq \int_{\Omega}\left(k_{7} u^{2 q-2}+k_{8}\right)|\nabla u|^{2} d x \\
& \leq \frac{k_{7}}{2} \int_{\Omega} u^{4 q-4} d x+\frac{k_{7}}{2} \int_{\Omega}|\nabla u|^{4} d x+k_{8}\|\nabla u\|^{2} \\
& \leq \frac{k_{7}}{2}\|\nabla u\|_{4}^{4}+\frac{k_{7} C}{2}+\frac{2 C_{6} C_{1}^{\prime} k_{8}}{\gamma} .
\end{aligned}
$$

Using Nirenberg-Gagliardo's inequality, we have

$$
\begin{aligned}
& \|\nabla u\|_{4} \leq C_{1}^{\prime}\left\|\Delta^{2} u\right\|^{\frac{n}{12}}\|\nabla u\|^{1-\frac{n}{12}}+C_{2}^{\prime}\|\nabla u\|(n \leq 2), \\
& \|\nabla u\|_{6} \leq C_{1}^{\prime}\left\|\Delta^{2} u\right\|^{\frac{n}{9}}\|\nabla u\|^{1-\frac{n}{9}}+C_{2}^{\prime}\|\nabla u\|(n \leq 2), \\
& \|\Delta u\|_{6} \leq C_{1}^{\prime}\left\|\Delta^{2} u\right\|^{\frac{3+n}{9}}\|\nabla u\|^{1-\frac{3+n}{9}}+C_{2}^{\prime}\|\nabla u\|(n \leq 2) .
\end{aligned}
$$

Using Young's inequality, we have

$$
\begin{aligned}
& \|\nabla u\|_{4}^{4} \leq C_{3}^{\prime}\left\|\Delta^{2} u\right\|^{\frac{2}{3}}+C_{4}^{\prime} \leq \varepsilon\left\|\Delta^{2} u\right\|^{2}+C_{\varepsilon}, \\
& \|\nabla u\|_{6}^{4} \leq C_{3}^{\prime}\left\|\Delta^{2} u\right\|^{\frac{8}{9}}+C_{4}^{\prime} \leq \varepsilon\left\|\Delta^{2} u\right\|^{2}+C_{\varepsilon}, \\
& \|\Delta u\|_{6}^{2} \leq C_{3}^{\prime}\left\|\Delta^{2} u\right\|^{\frac{10}{9}}+C_{4}^{\prime} \leq \varepsilon\left\|\Delta^{2} u\right\|^{2}+C_{\varepsilon} .
\end{aligned}
$$

Thus, we deduce that

$$
\begin{aligned}
\frac{d}{d t}\|\Delta u\|^{2}+\gamma\left\|\Delta^{2} u\right\|^{2} \leq & 2 C_{7} M_{1}\left(\|\Delta u\|_{6}^{2}+\|\nabla u\|_{6}^{4}\right) \\
& +2 C_{8}\left(\frac{k_{7} C}{2}+\frac{2 C_{6} C_{1}^{\prime} k_{8}}{\gamma}+\frac{k_{7}}{2}\|\nabla u\|_{4}^{4}\right) \\
\leq & 2 C_{7} M_{1}\left(2 \varepsilon\left\|\Delta^{2} u\right\|^{2}+2 C_{\varepsilon}\right) \\
& +2 C_{8}\left(\frac{k_{7} C}{2}+\frac{2 C_{6} C_{1}^{\prime} k_{8}}{\gamma}+\frac{k_{7} \varepsilon}{2}\left\|\Delta^{2} u\right\|^{2}+\frac{k_{7} C_{\varepsilon}}{2}\right) .
\end{aligned}
$$

Therefore

$$
\frac{d}{d t}\|\Delta u\|^{2}+\left(\gamma-4 C_{7} M_{1} \varepsilon-C_{8} k_{7} \varepsilon\right)\left\|\Delta^{2} u\right\|^{2} \leq C_{9},
$$


where $C_{9}=4 C_{7} M_{1} C_{\varepsilon}+C_{8} k_{7} C+\frac{4}{\gamma} C_{6} C_{1}^{\prime} C_{8} k_{8}+C_{8} k_{7} C_{\varepsilon}>0$. Taking $\varepsilon$ small enough, for $C_{10}>0$ and $C_{11}>0$, we obtain

$$
\frac{d}{d t}\|\Delta u\|^{2}+C_{10}\left\|\Delta^{2} u\right\|^{2} \leq C_{11} .
$$

By the Calderon-Zygmund type estimate, we have

$$
\frac{d}{d t}\|\Delta u\|^{2}+C^{\prime} C_{10}\left(\|\Delta u\|^{2}+\|\nabla \Delta u\|^{2}\right) \leq C_{11} .
$$

By Gronwall's inequality and $\|\Delta u(0)\| \leq R$, there holds

$$
\begin{aligned}
\|\Delta u\|^{2} & \leq e^{-C^{\prime} C_{10} t}\left\|\Delta u_{0}\right\|^{2}+\frac{C_{11}}{C^{\prime} C_{10}} \\
& \leq e^{-C^{\prime} C_{10} t} R^{2}+\frac{C_{11}}{C^{\prime} C_{10}} \leq \frac{2 C_{11}}{C^{\prime} C_{10}}
\end{aligned}
$$

for $t \geq t_{3}(B)=\frac{1}{C^{\prime} C_{10}} \ln \frac{C^{\prime} C_{10} R^{2}}{C_{11}}$. Adding (2.26) and (2.33) together, we obtain

$$
\|u(t)\|_{H^{2}} \leq C \text {. }
$$

Based on Sobolev's embedding theorem, we obtain

$$
\|u(t)\|_{L^{\infty}} \leq C \text {. }
$$

Let $t_{0}(B)=\max \left\{t_{1}(B), t_{2}(B), t_{3}(B)\right\}$, the lemma is proved.

The above lemma implies that the operator semigroup $\{S(t)\}_{t \geq 0}$ has a bounded absorbing set in $\mathscr{U}$. In the following Lemma 2.4, the precompactness of the orbit in $\mathscr{U}$ is proved.

Lemma 2.4. For initial data $u_{0}$ varying in a bounded set $B \subset \mathscr{U}$, there exists a $t_{1}(B)>0$ such that

$$
\|u(t)\|_{H^{3}} \leq C, \quad \forall t \geq t_{1}>0,
$$

which turns out that $\bigcup_{t \geq t_{1}} u(t)$ is relatively compact in $\mathscr{U}$.

Proof. The uniform bound of $H^{2}(\Omega)$ norm of $u(t)$ has been obtained in the above lemma. In the following we derive the estimate on $H^{3}$ norm. Acting $\Delta$ on the equation (1.1), we obtain

$$
\frac{\partial \Delta u}{\partial t}+\gamma \Delta^{3} u=\Delta^{2} A(u)+\beta \cdot \nabla \Delta B(u),
$$

and when $x \in \partial \Omega$, the following (2.37) is completed

$$
\Delta u=\Delta^{2} u \text {. }
$$

Multiplying (2.36) by $\Delta^{2} u$ and integrating the result over $\Omega$, we immediately deduce that

$$
\begin{aligned}
& \frac{1}{2} \frac{d}{d t}\|\nabla \Delta u\|^{2}+\gamma \int_{\Omega}\left|\nabla \Delta^{2} u\right|^{2} d x \\
& -\int_{\Omega} \nabla \Delta A(u) \nabla \Delta^{2} u d x-\beta \cdot \int_{\Omega} \Delta B(u) \nabla \Delta^{2} u d x=0 .
\end{aligned}
$$


On the other hand, we have

$$
\nabla \Delta A(u)=\nabla\left(A^{\prime}(u) \Delta u+A^{\prime \prime}(u)|\nabla u|^{2}\right),
$$

and

$$
\Delta B(u)=\nabla\left(B^{\prime}(u) \nabla u\right) .
$$

Hence, a simple conclusion shows that

$$
\begin{aligned}
& \left|\int_{\Omega} \nabla \Delta A(u) \cdot \nabla \Delta^{2} u d x\right| \\
= & \left|\int_{\Omega} \nabla\left(A^{\prime}(u) \Delta u+A^{\prime \prime}(u)|\nabla u|^{2}\right) \nabla \Delta^{2} u d x\right| \\
\leq & \left(\int_{\Omega}\left|A^{\prime}(u) \nabla \Delta u \nabla \Delta^{2} u\right| d x+3 \int_{\Omega}\left|A^{\prime \prime}(u) \nabla u \Delta u \nabla \Delta^{2} u\right| d x\right. \\
& \left.+\int_{\Omega}\left|A^{\prime \prime \prime}(u)(\nabla u)^{3} \nabla \Delta^{2} u\right| d x\right) \\
\leq & C\left(\int_{\Omega}\left|\nabla \Delta u \nabla \Delta^{2} u\right| d x+3 \int_{\Omega}\left|\nabla u \Delta u \nabla \Delta^{2} u\right| d x\right. \\
& \left.+\int_{\Omega}\left|(\nabla u)^{3} \nabla \Delta^{2} u\right| d x\right) \\
\leq & C_{\varepsilon}\left(\|\nabla \Delta u\|^{2}+\|\nabla u \Delta u\|^{2}+\|\nabla u\|_{6}^{6}\right)+\varepsilon\left\|\nabla \Delta^{2} u\right\|^{2},
\end{aligned}
$$

and

$$
\begin{aligned}
& \left|\int_{\Omega} \Delta B(u) \nabla \Delta^{2} u d x\right| \\
= & \left|\int_{\Omega} \nabla\left(B^{\prime}(u) \nabla u\right) \nabla \Delta^{2} u d x\right| \\
\leq & \int_{\Omega}\left|B^{\prime}(u) \Delta u \nabla \Delta^{2} u\right| d x+\int_{\Omega}\left|B^{\prime \prime}(u)(\nabla u)^{2}\right| \nabla \Delta^{2} u \mid d x \\
\leq & C\left(\int_{\Omega}\left|\Delta u \nabla \Delta^{2} u\right| d x+\int_{\Omega}|\nabla u|^{2}\left|\nabla \Delta^{2} u\right| d x\right) \\
\leq & C_{\varepsilon}\left(\|\Delta u\|^{2}+\|\nabla u\|_{4}^{4}\right)+\varepsilon\left\|\nabla \Delta^{2} u\right\|^{2} .
\end{aligned}
$$

Based on Nirenberg's inequality, we obtain

(2.41) $\|\nabla u\|_{4}^{4} \leq C\left(\left\|\nabla \Delta^{2} u\right\|^{\frac{n}{4}}\|\nabla u\|^{4-\frac{n}{4}}+\|\nabla u\|^{4}\right) \leq \varepsilon\left\|\nabla \Delta^{2} u\right\|^{2}+C_{\varepsilon}(n \leq 2)$, and we also have the following inequality

$$
\|\nabla u \Delta u\|^{2} \leq C\|\nabla u\|_{L^{\infty}}^{2}\|\Delta u\|^{2} \leq C_{12}\|\nabla u\|_{L^{\infty}}^{2} .
$$

By Nirenberg's inequality again, we deduce that

(2.43) $\|\nabla u\|_{\infty}^{2} \leq C\left(\left\|\nabla \Delta^{2} u\right\|^{\frac{n}{4}}\|\nabla u\|^{2-\frac{n}{4}}+\|\nabla u\|^{2}\right) \leq \varepsilon\left\|\nabla \Delta^{2} u\right\|^{2}+C_{\varepsilon}(n \leq 2)$, and we also have

(2.44) $\|\nabla u\|_{6}^{6} \leq C\left(\left\|\nabla \Delta^{2} u\right\|^{\frac{n}{2}}\|\nabla u\|^{6-\frac{n}{2}}+\|\nabla u\|^{6}\right) \leq \varepsilon\left\|\nabla \Delta^{2} u\right\|^{2}+C_{\varepsilon}(n \leq 2)$. 
Using (2.38)-(2.44), we obtain

$$
\begin{aligned}
& \frac{1}{2} \frac{d}{d t}\|\nabla \Delta u\|^{2}+\left(\gamma-\left(C_{12} C_{\varepsilon}+C_{\varepsilon}+1+|\beta| C_{\varepsilon}+|\beta|\right) \varepsilon\right)\left\|\nabla \Delta^{2} u\right\|^{2} \\
\leq & C_{\varepsilon}\|\nabla \Delta u\|^{2}+C_{13},
\end{aligned}
$$

where

$$
C_{13}=C_{12} C_{\varepsilon}^{2}+C_{\varepsilon}^{2}+|\beta| C_{\varepsilon}^{2}+\frac{2|\beta| C_{11} C_{\varepsilon}}{C^{\prime} C_{10}}>0 .
$$

Taking $\varepsilon$ small enough and $\gamma$ satisfies $\gamma-\left(C_{12} C_{\varepsilon}+C_{\varepsilon}+1+|\beta| C_{\varepsilon}+|\beta|\right) \varepsilon>0$, using Young's inequality, we have

$$
\frac{1}{2} \frac{d}{d t}\|\nabla \Delta u\|^{2} \leq C_{\varepsilon}\|\nabla \Delta u\|^{2}+C_{13} .
$$

On the other hand, integrating (2.32) between $t$ and $t+1$, we immediately conclude that

$$
C^{\prime} C_{10} \int_{t}^{t+1}\|\nabla \Delta u\|^{2} d \tau \leq\|\Delta u(t)\|^{2}+C_{11} \leq C_{11}\left(\frac{2}{C^{\prime} C_{10}}+1\right) .
$$

Owning to (2.45), (2.46) and the uniform Gronwall inequality inequality in [16], we deduce that

The lemma is proved.

$$
\|\nabla \Delta u\|^{2} \leq C, \quad t \geq 1
$$

Then by Theorem I.1.1 in [16], we immediately conclude that $\mathscr{A}=\omega(\mathscr{B})$. The $\omega$-limit set of absorbing set $\mathscr{B}$ is a global attractor in $\mathscr{U}$. By Lemma 2.4, this global attractor is a bounded set in $H^{3}(\Omega)$. Thus the proof of Theorem 2.1 is complete.

Remark 2.1. Because of the convective term, the convective Cahn-Hilliard equation is different from the usual Cahn-Hilliard equation in some properties. For the usual Cahn-Hilliard equation (i.e., $B(u)=0$ ) with following boundary conditions

$$
\frac{\partial u}{\partial n}=\frac{\partial \Delta u}{\partial n}, x \in \partial \Omega
$$

it is easy to obtain that the $L^{2}$ norm estimate and the $H^{1}$ norm estimate on $u$, since the usual Cahn-Hilliard equation with the above boundary conditions has two important properties:

(1) the conservation of mass, namely

$$
\int_{\Omega} u(x, t) d x=\int_{\Omega} u_{0}(x) d x
$$

(2) there exists a Lyapunov functional

$$
F(u)=\int_{\Omega}\left(\frac{\gamma}{2}|\nabla u|^{2}+H(u)\right) d x,
$$

which is decreasing in time. 
However, for problem (1.1)-(1.3), the above two properties may not be existent. So, we have to use another approach to establish the a prior estimates on $\|u\|_{L^{2}(\Omega)}$ and $\|u\|_{H^{1}(\Omega)}$ : Multiplying both sides of Eq.(1.1) by $u$ and $\Delta u$, integrating the result over $\Omega$. Since there's no useful method to avoid applying Nirenberg's inequality when the $H^{1}$ norm estimate is established, we have to confront some restricted conditions. We cannot help but consider this problem in one or two dimension space, and we have got to choose some condition for the order of polynomials $A(u)$ and $B(u)$, where $A(u)$ 's order satisfies $2 p-1 \leq \frac{4}{n}+1$ and $B(u)^{\prime}$ 's $q \leq \frac{6}{n}+1$.

\section{Attractor in $H^{k}$ space}

\subsection{Preliminary}

In this part, we used to assume that the linear operator $L: X_{1} \rightarrow X$ in (2.1) is a sectorial operator, which generates an analytic semigroup $e^{t L}$, and $L$ induces the fractional power operators and fractional order spaces as follows

$$
\mathscr{L}^{\alpha}=(-L)^{\alpha}: X_{\alpha} \rightarrow X, \alpha \in \mathbb{R},
$$

where $X_{\alpha}=D\left(\mathscr{L}^{\alpha}\right)$ is the domain of $\mathscr{L}^{\alpha}$. By the semigroup theory of linear operators, $X_{\beta} \subset X_{\alpha}$ is a compact inclusion for any $\beta>\alpha$. For more details on the space $H_{\alpha}$, see the reference [10].

Thus, Lemma 2.1 can be equivalently expressed in the following Lemma 3.1. We can find Lemma 3.1 in $[13,14,15]$.

Lemma 3.1. Assume that $u(t, \varphi)=S(t) \varphi(\varphi \in X, t \geq 0)$ is a solution of problem (2.1) and $S(t)$ the semigroup generated by problem (2.1). Assume further that $X_{\alpha}$ is the fractional order space generated by $L$ and

(B1) For some $\alpha \geq 0$ there is a bounded set $B \subset X_{\alpha}$, which means for any $\varphi \in X_{\alpha}$, there exists $t_{\varphi} \geq 0$ such that

$$
u(t, \varphi) \in B, \forall t>t_{\varphi},
$$

(B2) There is a $\beta>\alpha$, for any bounded set $U \subset X_{\beta}$, there are $T>0$ and $C>0$ such that

$$
\|u(t, \varphi)\|_{X_{\beta}} \leq C, \forall t \geq T, \varphi \in U .
$$

Then (2.1) has a global attractor $\mathscr{A} \subset X_{\alpha}$ which attracts any bounded set of $X_{\alpha}$ in the $X_{\alpha}$-norm.

We also have the following lemma which can be founded in $[13,14,15]$.

Lemma 3.2. Assume that $L: X_{1} \rightarrow X_{\alpha}$ is a sectorial operator which generates an analytic semigroup $T(t)=e^{t L}$. If all eigenvalues $\lambda$ of $L$ satisfy $\operatorname{Re} \lambda<-\lambda_{0}$ for some real number $\lambda_{0}>0$, then for $\mathscr{L}^{\alpha}(\mathscr{L}=-L)$ we have

(C1) $T(t): X \rightarrow X_{\alpha}$ is bounded for all $\alpha \in R^{1}$ and $t>0$;

(C2) $T(t) \mathscr{L}^{\alpha} x=\mathscr{L} T(t) x, \forall x \in X_{\alpha}$; 
(C3) For each $t>0, \mathscr{L}^{\alpha} T(t): X \rightarrow X$ is bounded, and

$$
\left\|\mathscr{L}^{\alpha} T(t)\right\| \leq C_{\alpha} t^{-\alpha} e^{-\delta t},
$$

where some $\delta>0$ and $C_{\alpha}>0$ is a constant depending only on $\alpha$;

(C4) The $X_{\alpha}$-norm can be defined by $\|x\|_{X_{\alpha}}=\left\|\mathscr{L}^{\alpha} x\right\|_{X}$.

For problem (1.1)-(1.3), we introduce the following space:

$$
\begin{cases}H & =L^{2}(\Omega), \\ H_{1 / 2} & =\left\{u \in H^{2}(\Omega) \bigcap H,\left.u\right|_{\partial \Omega}=0\right\}, \\ H_{1} & =\left\{H^{4}(\Omega) \bigcap H,\left.u\right|_{\partial \Omega}=\left.\Delta u\right|_{\partial \Omega}=0\right\},\end{cases}
$$

where $\Omega$ is a bounded domain in $\mathbb{R}^{n}(n \leq 2)$. We define the linear operators $L: H_{1} \rightarrow H$ and $G: H_{1} \rightarrow H$ by

$$
\begin{cases}L u & =-\Delta^{2} u, \\ g(u) & =\Delta A(u)+\beta \cdot \nabla B(u), \\ G(u) & =g(u) .\end{cases}
$$

It is easy to check that $L$ given by (3.2) is a sectorial operator and the tractional power operator $(-L)^{1 / 2}$ is given by

$$
(-L)^{1 / 2}=-\Delta \text {. }
$$

The space $H_{1 / 2}$ is the same as (3.1), and $H_{1 / 4}$ is given by $H_{1 / 4}=$ closure of $H_{1 / 2}$ in $H^{1}(\Omega)$ and $H_{k}=H^{4 k} \bigcap H_{1}$ for $k \geq 1$.

The main result is given by the following theorem, which provides the existence of global attractors of the convective Cahn-Hilliard equation in any $k$ th space $H^{k}$.

Theorem 3.1. Assume $\Omega \subset \mathbb{R}^{2}$ is a bounded domain. Then for any $\alpha>0$, problem (1.1)-(1.3) possesses a global attractor $\mathscr{A}$ in $H_{\alpha}$ space and $\mathscr{A}$ attractors any bounded set of $H_{\alpha}$ in the $H_{\alpha}$-norm.

\subsection{Proof of Theorem 3.1}

It's well known that the solution $u\left(t, u_{0}\right)$ of problem (1.1)-(1.3) can be written as

$$
u\left(t, u_{0}\right)=e^{t L} u_{0}+\int_{0}^{t} e^{(t-\tau) L} G(u) d \tau .
$$

Using (3.2) and (3.3), we obtain

$$
u\left(t, u_{0}\right)=e^{t L} u_{0}+\int_{0}^{t} e^{(t-\tau) L} g(u) d \tau .
$$

Based on Lemma 3.1, In order to prove Theorem 3.1, we first prove the following lemma.

Lemma 3.3. Assume $\Omega \subset \mathbb{R}^{2}$, then for any $\alpha \geq 0$, the semigroup $S(t)$ generated by the problem (1.1)-(1.3) is uniformly compact in $H_{\alpha}$. 
Proof. It suffices to prove that for any bounded set $U \subset H_{\alpha}$, there exists $C>0$ such that

$$
\left\|u\left(t, u_{0}\right)\right\|_{H_{\alpha}} \leq C, \quad \forall t \geq 0, u_{0} \in U \subset H_{\alpha}, \alpha \geq 0 .
$$

For $\alpha=\frac{1}{2}$, this follows form Theorem 2.1, i.e., for any bounded set $U \subset H_{1 / 2}$, there is a constant $C>0$ such that

$$
\left\|u\left(t, u_{0}\right)\right\|_{H_{1 / 2}} \leq C, \quad \forall t \geq 0, u_{0} \in U \subset H_{1 / 2} .
$$

Thus, we shall prove (3.5) for any $\alpha>\frac{1}{2}$. The lemma will be proved in the following steps.

First, we prove that for any bounded set $U \subset H_{\alpha}\left(\frac{1}{2} \leq \alpha<1\right)$, there exists a constant $C>0$ such that

$$
\left\|u\left(t, u_{0}\right)\right\|_{H_{\alpha}} \leq C, \quad \forall t \geq 0, u_{0} \in U, \alpha<1 .
$$

Based on the embedding theorems, we derive that

$$
H_{1 / 2} \hookrightarrow W^{1,2}(\Omega), \quad H_{1 / 2} \hookrightarrow W^{1,4}(\Omega) .
$$

Using (2.35) and the above embedding, we obtain

$$
\begin{aligned}
\|g(u)\|_{H}^{2}= & \int_{\Omega}|g(u)|^{2} d x \\
= & \left.\int_{\Omega}\left|A^{\prime \prime}(u)\right| \nabla u\right|^{2}+A^{\prime}(u) \Delta u+\left.\beta \cdot\left(B^{\prime}(u) \nabla u\right)\right|^{2} d x \\
\leq & \left.C \int_{\Omega}\left|A^{\prime \prime}(u)^{2}\right| \nabla u\right|^{4}+A^{\prime}(u)^{2}(\Delta u)^{2}+\left.\left(B^{\prime}(u)^{2}|\nabla u|^{2}\right)\right|^{2} d x \\
\leq & C\left(\sup _{x \in \Omega} A^{\prime \prime}(u)^{2} \int_{\omega}|\nabla u|^{4} d x+\sup _{x \in \Omega} A^{\prime}(u)^{2} \int_{\Omega}(\Delta u)^{2} d x\right. \\
& \left.+\sup _{x \in \Omega} B^{\prime}(u)^{2} \int_{\Omega}|\nabla u|^{2} d x\right) \\
\leq & C\left(\int_{\Omega}|\nabla u|^{4} d x+\int_{\Omega}|\Delta u|^{2} d x+\int_{\Omega}|\nabla u|^{2} d x\right) \\
\leq & C\left(\|u\|_{W^{1,4}}^{4}+\|u\|_{H_{1 / 2}}^{2}+\|u\|_{W^{1,2}}^{2}\right) \\
\leq & C\left(\|u\|_{H_{1 / 2}}^{4}+\|u\|_{H_{1 / 2}}^{2}+\|u\|_{H_{1 / 2}}^{2}\right),
\end{aligned}
$$

which means that $g: H_{1 / 2} \rightarrow H$ is bounded.

Hence, we deduce that

$$
\begin{aligned}
\left\|u\left(t, u_{0}\right)\right\|_{H_{\alpha}} & =\left\|e^{t L} u_{0}+\int_{0}^{t} e^{(t-\tau) L} g(u) d \tau\right\|_{H_{\alpha}} \\
& \leq C\left\|u_{0}\right\|_{H_{\alpha}}+\int_{0}^{t}\left\|(-L)^{\alpha} e^{(t-\tau) L} g(u)\right\|_{H} d \tau \\
& \leq C\left\|u_{0}\right\|_{H_{\alpha}}+\int_{0}^{t}\left\|(-L)^{\alpha} e^{(t-\tau) L}\right\| \cdot\|g(u)\|_{H} d \tau
\end{aligned}
$$




$$
\begin{aligned}
& \leq C\left\|u_{0}\right\|_{H_{\alpha}}+C \int_{0}^{t} \tau^{-\alpha} e^{-\delta \tau} d \tau \\
& \leq C, \quad \forall t \geq 0, u_{0} \in U \subset H_{\alpha},
\end{aligned}
$$

where $0<\alpha<1$.

Thus, (3.7) is proved.

Second, we prove that for any bounded set $U \subset H_{\alpha}\left(1 \leq \alpha<\frac{5}{4}\right)$, there exists a constant $C>0$, such that

$$
\left\|u\left(t, u_{0}\right)\right\|_{H_{\alpha}} \leq C, \quad \forall t \geq 0, u_{0} \in U \subset H_{\alpha}, 1 \leq \alpha<\frac{5}{4}
$$

Based on the following embedding theorems of fractional order spaces (see Pazy [11]), we derive that

$$
\begin{array}{ll}
H_{\alpha} \hookrightarrow W^{3,2}(\Omega), & H_{\alpha} \hookrightarrow W^{2,4}(\Omega), \\
H_{\alpha} \hookrightarrow W^{1,6}(\Omega), & H_{\alpha} \hookrightarrow W^{1,4}(\Omega),
\end{array}
$$

where $\frac{3}{4} \leq \alpha<1$. Then, using (2.35), we obtain

$$
\begin{aligned}
& \|g(u)\|_{H_{1 / 4}}^{2} \\
= & \int_{\Omega}(\nabla(\Delta A(u)+\beta \cdot \nabla B(u)))^{2} d x \\
= & \int_{\Omega}\left(A^{\prime \prime \prime}(u)|\nabla u|^{3}+3 A^{\prime \prime}(u)|\nabla u \Delta u|+A^{\prime}(u)|\nabla \Delta u|\right. \\
& \left.+B^{\prime \prime}(u)|\beta| \cdot|\nabla u|^{2}+|\beta| B^{\prime}(u) \Delta u\right)^{2} d x \\
\leq & C\left(\left.\int_{\Omega}\left|A^{\prime \prime \prime}(u)^{2}\right| \nabla u\right|^{6} d x+\left.\int_{\Omega}\left|A^{\prime \prime}(u)^{2}\right| \nabla u \Delta u\right|^{2} \mid d x\right. \\
& \left.+\left.\int_{\Omega}\left|A^{\prime}(u)^{2}\right| \nabla \Delta u\right|^{2} d x+\left.\int_{\Omega}\left|B^{\prime \prime}(u)^{2}\right| \nabla u\right|^{4} d x+\int_{\Omega} \mid B^{\prime}(u)^{2}(\Delta u)^{2} d x\right) \\
\leq & C\left(\sup _{x \in \Omega} A^{\prime \prime \prime}(u)^{2} \int_{\Omega}|\nabla u|^{6} d x+\sup _{x \in \Omega} A^{\prime \prime}(u)^{2} \int_{\Omega}\left(|\nabla u|^{4}+(\Delta u)^{4}\right) d x\right. \\
& \left.+\sup _{x \in \Omega} A^{\prime}(u)^{2} \int_{\Omega}(\nabla \Delta u)^{2} d x+\sup _{x \in \Omega} B^{\prime \prime}(u) \int_{\Omega}|\nabla u|^{4} d x+\sup _{x \in \Omega}(\Delta u)_{\Omega}^{2} d x\right) \\
\leq & C\left(\|u\|_{W^{1,6}}^{6}+\|u\|_{W^{1,4}}^{4}+\|u\|_{W^{2,4}}^{2}+\|u\|_{W^{3,2}}^{2}+\|u\|_{H_{1 / 2}}^{4}\right) \\
\leq & C\left(\|u\|_{H_{\alpha}}^{6}+\|u\|_{H_{\alpha}}^{2}+\|u\|_{H_{\alpha}}^{4}\right),
\end{aligned}
$$

which means that $g: H_{\alpha} \rightarrow H_{1 / 4}$ is bounded for $\frac{3}{4} \leq \alpha<1$. Using (3.7) and (3.11), we obtain

$$
\left\|g\left(u\left(t, u_{0}\right)\right)\right\|_{H_{1 / 4}} \leq C, \quad \forall t \geq 0, \varphi \in U, \frac{3}{4} \leq \alpha<1 .
$$


By using the same method as that in the first step, from (3.12), we derive that

$$
\begin{aligned}
\left\|u\left(t, u_{0}\right)\right\|_{H_{\alpha}} & =\left\|e^{t L} u_{0}+\int_{0}^{t} e^{(t-\tau) L} g(u) d \tau\right\|_{H_{\alpha}} \\
& \leq C\left\|u_{0}\right\|_{H_{\alpha}}+\int_{0}^{t}\left\|(-L)^{\alpha} e^{(t-\tau) L} g(u)\right\|_{H} d \tau \\
& \leq C\left\|u_{0}\right\|_{H_{\alpha}}+\int_{0}^{t}\left\|(-L)^{\alpha-\frac{1}{4}} e^{(t-\tau) L}\right\| \cdot\|g(u)\|_{H_{1 / 4}} d \tau \\
& \leq C\left\|u_{0}\right\|_{H_{\alpha}}+C \int_{0}^{t} \tau^{-\beta} e^{-\delta \tau} d \tau \\
& \leq C, \quad \forall t \geq 0, u_{0} \in U \subset H_{\alpha},
\end{aligned}
$$

where $\beta=\alpha-\frac{1}{4}(0<\beta<1)$.

Thus (3.10) is proved.

Third, we prove that for any bounded set $U \subset H_{\alpha}\left(\frac{5}{4} \leq \alpha<\frac{3}{2}\right)$ there exists a constant $C>0$, such that

$$
\left\|u\left(t, u_{0}\right)\right\|_{H_{\alpha}} \leq C, \quad \forall t \geq 0, u_{0} \in U \subset H_{\alpha}, \frac{5}{4} \leq \alpha<\frac{3}{2} .
$$

Based on the following embedding theorems of fractional order spaces (see Pazy [11]), we deduce that

$$
\begin{aligned}
& H_{\alpha} \hookrightarrow W^{1,4}(\Omega), \quad H_{\alpha} \hookrightarrow W^{2,4}(\Omega), \quad H_{\alpha} \hookrightarrow W^{3,4}(\Omega), \\
& H_{\alpha} \hookrightarrow W^{4,2}(\Omega), \quad H_{\alpha} \hookrightarrow W^{3,2}(\Omega), \quad H_{\alpha} \hookrightarrow W^{1,8}(\Omega), \\
& H_{\alpha} \hookrightarrow W^{1,6}(\Omega),
\end{aligned}
$$

where $1 \leq \alpha<\frac{5}{4}$. Then, using (2.35), we obtain

$$
\begin{aligned}
& \|g(u)\|_{H_{1 / 2}}^{2} \\
= & \int_{\Omega}|\Delta(\Delta A(u)+\beta \cdot \nabla B(u))|^{2} d x \\
\leq & \int_{\Omega}\left(A^{(4)}(u)|\nabla u|^{4}+6 A^{\prime \prime \prime}(u)|\nabla u|^{2} \Delta u+3 A^{\prime \prime}(u)(\Delta u)^{2}\right. \\
& +4 A^{\prime \prime}(u)|\nabla u \nabla \Delta u|+A^{\prime}(u) \Delta^{2} u+B^{\prime \prime \prime}(u)|\beta| \cdot|\nabla u|^{3} \\
& \left.+3 B^{\prime \prime}(u)|\beta| \cdot|\nabla u \Delta u|+B^{\prime}(u)|\beta| \cdot|\nabla \Delta u|\right)^{2} d x \\
\leq & C\left(\int_{\Omega}\left(A^{(4)}(u)\right)^{2}|\nabla u|^{8} d x+\int_{\Omega} A^{\prime \prime \prime}(u)^{2}|\nabla u|^{4}(\Delta u)^{2} d x\right. \\
& +\int_{\Omega} A^{\prime \prime}(u)^{2}(\Delta u)^{4} d x+\int_{\Omega} A^{\prime \prime}(u)^{2}|\nabla u|^{2}|\nabla \Delta u|^{2} d x \\
& +\int_{\Omega} A^{\prime}(u)^{2}\left(\Delta^{2} u\right)^{2} d x+\int_{\Omega} B^{\prime \prime \prime}(u)^{2}|\nabla u|^{6} d x+\int_{\Omega} B^{\prime \prime}(u)|\nabla u \Delta u|^{2} d x
\end{aligned}
$$




$$
\begin{aligned}
& \left.+\int_{\Omega} B^{\prime}(u)^{2}|\nabla \Delta u|^{2} d x\right) \\
\leq & C\left(\operatorname { s u p } _ { x \in \Omega } \left(A^{(4)}(u)^{2} \int_{\Omega}|\nabla u|^{8} d x+\sup _{x \in \Omega}\left(A^{\prime \prime \prime}(u)\right)^{2} \int_{\Omega}\left(\left.\nabla u\right|^{8}+|\Delta u|^{4}\right) d x\right.\right. \\
& +\sup _{x \in \Omega}\left(A^{\prime \prime}(u)\right)^{2} \int_{\Omega}(\Delta u)^{4} d x+\sup _{x \in \Omega}\left(A^{\prime \prime}(u)\right)^{2} \int_{\Omega}\left(|\nabla u|^{4}+|\nabla \Delta u|^{4}\right) d x \\
& +\sup _{x \in \Omega}\left(A^{\prime}(u)\right)^{2} \int_{\Omega}\left|\Delta^{2} u\right|^{2} d x+\sup _{x \in \Omega}\left(B^{\prime \prime \prime}(u)\right)^{2} \int_{\Omega}|\nabla u|^{6} d x \\
& \left.+\sup _{x \in \Omega}\left(B^{\prime \prime}(u)\right)^{2} \int_{\Omega}\left(|\nabla u|^{4}+|\Delta u|^{4}\right) d x+\sup _{x \in \Omega}\left(B^{\prime}(u)\right)^{2} \int_{\Omega}|\nabla \Delta u|^{2} d x\right) \\
\leq & C\left(\|u\|_{W^{1,8}}^{8}+\|u\|_{W^{2,4}}^{4}+\|u\|_{W^{3,4}}^{4}+\|u\|_{W^{4,2}}^{2}+\|u\|_{W^{1,6}}^{6}\right. \\
& \left.+\|u\|_{W^{3,2}}^{2}+\|u\|_{W^{1,4}}^{4}\right) \\
\leq & C\left(\|u\|_{H_{\alpha}}^{8}+\|u\|_{H_{\alpha}}^{4}+\|u\|_{H_{\alpha}}^{2}+\|u\|_{H_{\alpha}}^{6}\right),
\end{aligned}
$$

which means that $g: H_{\alpha} \rightarrow H_{1 / 2}$ is bounded for $1 \leq \alpha<\frac{5}{4}$. Using (3.10) and (3.15), we obtain

$$
\left\|g\left(u\left(t, u_{0}\right)\right)\right\|_{H_{1 / 2}} \leq C, \quad \forall t \geq 0, u_{0} \in U, 1 \leq \alpha<\frac{5}{4} .
$$

By using the same method as that in the first and second steps, from (3.16), we have

$$
\begin{aligned}
\left\|u\left(t, u_{0}\right)\right\|_{H_{\alpha}} & =\left\|e^{t L} u_{0}+\int_{0}^{t} e^{(t-\tau) L} g(u) d \tau\right\|_{H_{\alpha}} \\
& \leq C\left\|u_{0}\right\|_{H_{\alpha}}+\int_{0}^{t}\left\|(-L)^{\alpha-\frac{1}{2}} e^{(t-\tau) L}\right\| \cdot\|g(u)\|_{H_{1 / 2}} d \tau \\
& \leq C\left\|u_{0}\right\|_{H_{\alpha}}+C \int_{0}^{t} \tau^{-\beta} e^{-\delta \tau} d \tau \\
& \leq C, \quad \forall t \geq 0, u_{0} \in U \subset H_{\alpha},
\end{aligned}
$$

where $\beta=\alpha-\frac{1}{2}(0<\beta<1)$.

Thus (3.14) is proved.

Fourth, we prove that for any bounded set $U \subset H_{\alpha}\left(\frac{3}{2} \leq \alpha<\frac{7}{4}\right)$, there exists a constant $C>0$ such that

$$
\left\|u\left(t, u_{0}\right)\right\|_{H_{\alpha}} \leq C, \quad \forall t \geq 0, u_{0} \in U \subset H_{\alpha}, \frac{3}{2} \leq \alpha<\frac{7}{4} .
$$

Based on the following embedding theorems of fractional order spaces (see Pazy [11]), we deduce that

$$
\begin{aligned}
& H_{\alpha} \hookrightarrow W^{1,10}(\Omega), \quad H_{\alpha} \hookrightarrow W^{1,12}(\Omega), \quad H_{\alpha} \hookrightarrow W^{2,4}(\Omega), \\
& H_{\alpha} \hookrightarrow W^{1,4}(\Omega), \quad H_{\alpha} \hookrightarrow W^{2,8}(\Omega), \quad H_{\alpha} \hookrightarrow W^{1,8}(\Omega),
\end{aligned}
$$




$$
\begin{aligned}
& H_{\alpha} \hookrightarrow W^{4,4}(\Omega), \quad H_{\alpha} \hookrightarrow W^{3,4}(\Omega), \quad H_{\alpha} \hookrightarrow W^{5,2}(\Omega), \\
& H_{\alpha} \hookrightarrow W^{4,2}(\Omega),
\end{aligned}
$$

where $\frac{5}{4} \leq \alpha<\frac{3}{2}$. Then, using (2.35), we obtain,

(3.19) $\|g(u)\|_{H_{3 / 4}}^{2}$

$$
\begin{aligned}
= & \int_{\Omega} \mid \nabla \Delta\left(\Delta A(u)+\left.\beta \cdot \nabla B(u)\right|^{2} d x\right. \\
\leq & \int_{\Omega}\left(A^{(5)}(u)|\nabla u|^{5}+10 A^{(4)}(u)|\nabla u|^{3} \Delta u+15 A^{\prime \prime \prime}(u)|\nabla u|(\Delta u)^{2}\right. \\
& +10 A^{\prime \prime \prime}(u)|\nabla u|^{2}|\nabla \Delta u|+10 A^{\prime \prime}(u)|\Delta u \nabla \Delta u|+5 A^{\prime \prime}(u)\left|\nabla u \Delta^{2} u\right| \\
& +A^{\prime}(u) \nabla \Delta^{2} u+B^{(4)}(u)|\beta| \cdot|\nabla u|^{4}+6 B^{\prime \prime \prime}(u)|\beta| \cdot|\nabla u|^{2} \Delta u \\
& \left.+3 B^{\prime \prime}(u)|\beta| \cdot|\Delta u|^{2}+4 B^{\prime \prime}(u)|\beta| \cdot|\nabla u \nabla \Delta u|+|\beta| B^{\prime}(u) \Delta^{2} u\right)^{2} d x \\
\leq & C\left(\sup _{x \in \Omega}\left(A^{(5)}(u)\right)^{2} \int_{\Omega}|\nabla u|^{10} d x+\sup _{x \in \Omega}\left(A^{(4)}\right)^{2} \int_{\Omega}\left(|\nabla u|^{12}+|\Delta u|^{4}\right) d x\right. \\
& +\sup _{x \in \Omega}\left(A^{\prime \prime \prime}(u)\right)^{2} \int_{\Omega}\left(|\nabla u|^{4}+|\Delta u|^{8}\right) d x+\sup _{x \in \Omega}\left(A^{\prime \prime \prime}(u)\right)^{2} \int_{\Omega}|\nabla u|^{8} d x \\
& +\sup _{x \in \Omega}\left(A^{\prime \prime \prime}(u)\right)^{2} \int_{\Omega}|\nabla \Delta u|^{4} d x+\sup _{x \in \Omega}\left(A^{\prime \prime}(u)\right)^{2} \int_{\Omega}\left(|\Delta u|^{4}+|\nabla \Delta u|^{4}\right) d x \\
& +\sup _{x \in \Omega}\left(A^{\prime \prime}(u)\right)^{2} \int_{\Omega}\left(|\nabla u|^{4}+\left|\Delta^{2} u\right|^{4}\right) d x+\sup _{x \in \Omega}\left(A^{\prime}(u)\right)^{2} \int_{\Omega}\left|\nabla \Delta^{2} u\right|^{2} d x \\
& +\sup _{x \in \Omega}\left(B^{(4)}\right)^{2} \int_{\Omega}|\nabla u|^{8} d x+\sup _{x \in \Omega}\left(B^{\prime \prime \prime}(u)\right)^{2} \int_{\Omega}\left(|\nabla u|^{8}+|\Delta u|^{4}\right) d x \\
& +\sup _{x \in \Omega}\left(B^{\prime \prime}(u)\right)^{2} \int_{\Omega}|\Delta u|^{4} d x+\sup _{x \in \Omega}\left(B^{\prime \prime}(u)\right)^{2} \int_{\Omega}\left(|\nabla u|^{4}+|\nabla \Delta u|^{4}\right) d x \\
& \left.+\sup _{x \in \Omega}\left(B^{\prime}(u)\right)^{2} \int_{\Omega}\left(\Delta^{2} u\right)^{2} d x\right) \\
\leq & C\left(\|u\|_{W^{1,10}}^{10}+\|u\|_{W^{1,12}}^{12}+\|u\|_{W^{2,4}}^{4}+\|u\|_{W^{1,4}}^{4}+\|u\|_{W^{2,8}}^{8}+\|u\|_{W^{1,8}}^{8}\right. \\
& \left.+\|u\|_{W^{3,4}}^{4}+\|u\|_{W^{5,2}}^{2}+\|u\|_{W^{4,2}}^{2}\right) \\
\leq & C\left(\|u\|_{H_{\alpha}}^{2}+\|u\|_{H_{\alpha}}^{4}+\|u\|_{H_{\alpha}}^{8}+\|u\|_{H_{\alpha}}^{10}+\|u\|_{H_{\alpha}}^{12}\right),
\end{aligned}
$$

which means that $g: H_{\alpha} \rightarrow H_{3 / 4}$ is bounded for $\frac{5}{4} \leq \alpha<\frac{3}{2}$. Using (3.14) and (3.19), we obtain

$$
\left\|g\left(u\left(t, u_{0}\right)\right)\right\|_{H_{3 / 4}} \leq C, \quad \forall t \geq 0, u_{0} \in U, \frac{5}{4} \leq \alpha<\frac{3}{2} .
$$

By using the same method as that in the above steps and from (3.20), we have

$$
\left\|u\left(t, u_{0}\right)\right\|_{H_{\alpha}}=\left\|e^{t L} u_{0}+\int_{0}^{t} e^{(t-\tau) L} g(u) d \tau\right\|_{H_{\alpha}}
$$




$$
\begin{aligned}
& \leq C\left\|u_{0}\right\|_{H_{\alpha}}+\int_{0}^{t}\left\|(-L)^{\alpha-\frac{3}{4}} e^{(t-\tau) L}\right\| \cdot\|g(u)\|_{H_{3 / 4}} d \tau \\
& \leq C\left\|u_{0}\right\|_{H_{\alpha}}+C \int_{0}^{t} \tau^{-\beta} e^{-\delta \tau} d \tau \\
& \leq C, \quad \forall t \geq 0, u_{0} \in U \subset H_{\alpha},
\end{aligned}
$$

where $\beta=\alpha-\frac{3}{4}(0<\beta<1)$.

Thus, (3.18) is proved.

In the same method as in the proof of (3.18), by iteration we can prove that for any bounded set $U \subset H_{\alpha}(\alpha \geq 0)$ there exists a constant $C>0$ such that (3.6) hold, i.e., for all $\alpha \geq 0$ the semigroup $S(t)$ generated by problem (1.1)-(1.3) is uniformly compact in $H_{\alpha}$.

The lemma is proved.

Lemma 3.4. Assume $\Omega \subset \mathbb{R}^{3}, u_{0} \in H_{0}^{2}(\Omega)$. Then for any $\alpha \geq 0$, problem (1.1)-(1.3) has a bounded absorbing set in $H_{\alpha}$.

Proof. It suffices to prove that for any bounded set $U \subset H_{\alpha}(\alpha \geq 0)$, there exist $T>0$ and a constant $C>0$ independent of $u_{0}$, such that

$$
\left\|u\left(t, u_{0}\right)\right\|_{H_{\alpha}} \leq C, \quad \forall t \geq T, u_{0} \in U \subset H_{\alpha} .
$$

For $\alpha=\frac{1}{2}$, this follows from Theorem 2.1. So we shall prove (3.22) for any $\alpha>\frac{1}{2}$. We prove the lemma in the following steps:

First, we prove that for any $\frac{1}{2} \leq \alpha<1$, problem (1.1)-(1.3) has a bounded absorbing set in $H_{\alpha}$.

By (3.2), we deduce that

$$
u\left(t, u_{0}\right)=e^{(t-T) L} u\left(T, u_{0}\right)+\int_{T}^{t} e^{(t-T) L} g(u) d \tau .
$$

Assume $B$ is the bounded absorbing set of problem (1.1)-(1.3) and satisfy $B \subset H_{1 / 2}$, we also assume $T_{0}>0$ the time such that

$$
u\left(t, u_{0}\right) \in B, \quad \forall t>T_{0}, u_{0} \in U \subset H_{\alpha}, \alpha \geq \frac{1}{2} .
$$

It is easy to check that

$$
\left\|e^{t L}\right\| \leq C e^{-d \lambda_{1}^{2} t}
$$

where $\lambda_{1}>0$ is the first eigenvalue of the equation

$$
\begin{cases}-\Delta u & =\lambda u, \\ \left.u\right|_{\partial \Omega} & =0 .\end{cases}
$$

Thus, for any given $T>0$ and $u_{0} \in U \subset H_{\alpha}\left(\alpha \geq \frac{1}{2}\right)$, we deduce that

$$
\lim _{t \rightarrow \infty}\left\|e^{(t-T) L} u(T, \varphi)\right\|_{H_{\alpha}}=0 .
$$


Using (3.6), (3.21) and the assertion (C3) of Lemma 3.2, we derive that

$$
\begin{aligned}
\left\|u\left(t, u_{0}\right)\right\|_{H_{\alpha}} & \leq\left\|e^{\left(t-T_{0}\right) L} u\left(T_{0}, u_{0}\right)\right\|_{H_{\alpha}}+\int_{T_{0}}^{t}\left\|(-L)^{\alpha} e^{(t-T) L}\right\| \cdot\|g(u)\|_{H} d \tau \\
& \leq\left\|e^{\left(t-T_{0}\right) L} u\left(T_{0}, u_{0}\right)\right\|_{H_{\alpha}}+C \int_{T_{0}}^{t}\left\|(-L)^{\alpha} e^{(t-T) L}\right\| \\
& \leq\left\|e^{\left(t-T_{0}\right) L} u\left(T_{0}, u_{0}\right)\right\|_{H_{\alpha}}+C \int_{0}^{T-T_{0}} \tau^{-\alpha} e^{-\delta \tau} d \tau \\
& \leq\left\|e^{\left(t-T_{0}\right) L} u\left(T_{0}, u_{0}\right)\right\|_{H_{\alpha}}+C
\end{aligned}
$$

where $C>0$ is a constant independent of $u_{0}$.

Then, by (3.25) and (3.26), we obtain (3.20) holds for all $\frac{1}{2} \leq \alpha<1$.

Second, we use the same method as the above step to prove that for any $\frac{3}{4}<\alpha<\frac{5}{4}$ and for any $1<\alpha<\frac{3}{2}$, the problem (1.1)-(1.3) has a bounded absorbing set in $H_{\alpha}$. By the iteration method, we obtain that (3.20) holds for all $\alpha \geq \frac{1}{2}$.

Hence, by Lemma 3.1, Lemma 3.3 and Lemma 3.4, we immediately conclude that the proof of Theorem 3.1 is completed.

Remark 3.1. The attractors $\mathscr{A}_{\alpha} \subset H_{\alpha}$ in Theorem 3.1 are the same for all $\alpha \geq 0$, i.e., $\mathscr{A}_{\alpha}=\mathscr{A}, \forall \alpha \geq 0$. Hence, $\mathscr{A} \subset C^{\infty}(\Omega)$. Theorem 3.1 implies that for any $u_{0} \in H$, the solution $u\left(t, u_{0}\right)$ of problem (1.1)-(1.3) satisfies that

$$
\lim _{t \rightarrow \infty} \inf _{v \in \mathscr{A}}\left\|u\left(t, u_{0}\right)-v\right\|_{C^{k}}=0, \quad \forall k \geq 1 .
$$

\section{References}

[1] J. W. Cholewa and T. Dlotko, Global attractor for the Cahn-Hilliard system, Bull. Austral. Math. Soc. 49 (1994), no. 2, 277-292.

[2] T. Dlotko, Global attractor for the Cahn-Hilliard equation in $H^{2}$ and $H^{3}$, J. Differential Equations 113 (1994), no. 2, 381-393.

[3] A. Eden and V. K. Kalantarov, The convective Cahn-Hilliard equation, Appl. Math. Lett. 20 (2007), no. 4, 455-461.

[4] - 3D convective Cahn-Hilliard equation, Commun. Pure Appl. Anal. 6 (2007), no. 4, 1075-1086

[5] A. A. Golovin, S. H. Davism, and A. A. Nepomnyashchy, A convective Cahn-Hilliard model for the formation of facets and corners in crystal growth, Phys. D 122 (1998), no. 1-4, 202-230.

[6] K. H. Kwek, On the Cahn-Hilliard type equation, Ph. D. thesis, Georgia Institute of Technology, 1991.

[7] D. Li and C. Zhong, Global attractor for the Cahn-Hilliard system with fast growing nonlinearity, J. Differential Equations 149 (1998), no. 2, 191-210.

[8] C. Liu, On the convective Cahn-Hilliard equation, Bull. Pol. Acad. Sci. Math. 53 (2005), no. $3,299-314$.

[9] _ On the convective Cahn-Hillirad equation with degenerate mobility, J. Math. Anal. Appl. 344 (2008), no. 1, 124-144. 
[10] T. Ma and S. H. Wang, Stability and Bifurcation of Nonlinear Evolution Equations, Science Press, Beijing, 2006.

[11] A. Pazy, Semigroups of linear operators and applications to partial differential equations, in: Appl. Math. Sci., vol. 44, Springer-Verlag, 1983.

[12] A. Podolny, M. A. Zaks, B. Y. Rubinstein, A. A. Golovin, and A. A. Nepomnyashchy, Dynamics of domain walls governed by the convective Cahn-Hilliard equation, Phys. D 201 (2005), no. 3-4, 291-305.

[13] L. Song, Y. He, and Y. Zhang, The existence of global attractors for semilinear parabolic equation in $H^{k}$ space, Nonlinear Anal. 68 (2008), no. 11, 3541-3549.

[14] L. Song, Y. Zhang, and T. Ma, Global attractor of the Cahn-Hilliard equation in $H^{k}$ spaces, J. Math. Anal. Appl. 355 (2009), no. 1, 53-62.

[15] _ Global attractor of a modified Swift-Hohenberg equation in $H^{k}$ space, Nonlinear Anal. 72 (2010), no. 1, 183-191.

[16] R. Temam, Infinite-Dimensional Dynamical Systems in Mechanics and Physics, Springer-Verlag, New York, 1988.

[17] S. J. Watson, F. Otto, B. Y. Rubinstein, and S. H. Davis, Coarsening dynamics of the convective Cahn-Hilliard equations, Phys. D 178 (2003), no. 3-4, 127-148.

[18] H. Wu and S. M. Zheng, Global attractor for the 1-D thin film equation, Asymptot. Anal. 51 (2007), no. 2, 101-111.

[19] M. A. Zarks, A. Podolny, A. A. Nepomnyashchy, and A. A. Golovin, Periodic stationary patterns governed by a convective Cahn-Hilliard equation, SIAM J. Appl. Math. 66 (2005), no. 2, 700-720.

[20] X. Zhao and C. Liu, Global attractor for the convective Cahn-Hilliard equation, Bull. Pol. Acad. Sci. Math. 58 (2010), no. 2, 117-127.

Xiaopeng Zhao

College of Mathematics

JILIN UNIVERSITY

Changchun, 130012, P. R. China

E-mail address: zxp032@gmail.com

Bo LiU

College of Mathematics

JiLIN UNIVERSITY

Changchun, 130012, P. R. China

E-mail address: liubom@jlu.edu.cn 